\title{
On the mathematical analysis of the limit case of a radiative - convective climate model
}

\author{
J.I. Díaz and J.I. Tello \\ Departamento de Matemática Aplicada, Facultad de CC. Matemáticas, \\ Universidad Complutense de Madrid, \\ 28040 Madrid, Spain
}

\begin{abstract}
We study the limit case corresponding to a model introduced by G.L. Stenchikov and A. Robock for the evolution of the temperature of an atmospheric column in ausence of humidity. The model envolves a degenerate non coercive quasilinear equation. The diffusion coefficient depends of the atmospheric stability and vanishes on the stable regions. We show the existence and uniqueness of a suitable class of weak solutions and prove that the number of stable and unstable regions are nonincreasing in time.
\end{abstract}

Keywords: Degenerate quasilinear equation; Meteorology; Number of stable regions.

\section{Introduction}

One of the simpler models in the very rich hierarchy of the climate models corresponds to the, so called, Radiative Convective Models (RCMs). This type of models was initially introduced in Manabe and Strickler [10] in order to describe averaged vertical atmospheric thermal structure when advective sources vanish due to spatial averaging. Since then, several modifications of the pioneers RCMs were introduce by several authors for different purposes (see e.g. the exposition made in Stenchikov and Robock [13]).

The main goal of this paper is to carry out the mathematical analysis of the limit case of a RCM introduced by G.L. Stenchikov and A. Robock in 1995 for the investigation of the diurnal cycle. Their model has a forced nature (by prescribed advection) and accounts interactively for boundary layer processes, turbulence, convection, cloudiness, and hydrological cycle, and incorporates a spectral radiative transport in a cloudy and polluted atmosphere. The model was used in Stenchikov and Robock [14] to calculate the effects of observed stratospheric aerosol, water vapor and ozone changes after the 1991 Pinatubo eruption.

For a given atmospheric column, let $H$ be the total specific energy, $T$ the temperature, $q$ the water vapor mixing ratio, $L$ the latent heat of vaporization, $c_{p}$ the specific 
heat of air at constant pressure, $H_{s}$ static energy, $g$ acceleration due to gravity, and $z$ is the altitude. Then $H=c_{p} T+L q$, and $H_{s}=H+g z$. In stable atmosphere regions, $\frac{\partial H}{\partial t}=0$, and in unstable regions the evolution of specific energy is given by $\frac{\partial H}{\partial t}=\frac{\partial}{\partial x} D \frac{\partial}{\partial x} H_{s}$, (see Stenchikov - Robock [13]), where the variable $x$ represents the normalized pressure.

Here we shall concern only with the dry case $q=0$. So $\frac{\partial}{\partial x} H_{s}=\frac{\partial H}{\partial x}-\frac{R T}{x}$, and the proposed model can be formulated in the following terms

$$
\begin{cases}\frac{\partial H}{\partial t}=\frac{\partial}{\partial x} k\left(\frac{\partial H}{\partial x}-\frac{R T}{x}\right), & x \in(0,1), t>0, \\ k\left(\frac{\partial H}{\partial x}-\frac{R T}{x}\right)=0, & x=0,1, t>0, \\ H(x, 0)=H_{0}(x), & x \in(0,1),\end{cases}
$$

where $R$ is a positive constant (the gas constant for the air), $c_{p} \in C^{0}([0,1]), c_{p}(x)>0$ on $(0,1)$ and the diffusion coefficient degenerates on $(-\infty, 0]$, more precisely

$$
k(s)= \begin{cases}k_{0} s & \text { if } s>0 \\ 0 & \text { otherwise }\end{cases}
$$

for $k_{0}=100 \frac{\varphi^{2} g^{2}}{P_{s}^{2}}$, where $g$ is the acceleration of the gravity and $\varphi$ is the density of the air. For simplicity we shall assume that $\varphi$ is constant. So, an equivalent formulated is

$$
\begin{cases}\left.\frac{\partial\left(c_{p} T\right)}{\partial t}=\frac{\partial}{\partial x} k\left(\frac{\partial\left(c_{p} T\right)}{\partial x}-\frac{R T}{x}\right)\right), & x \in(0,1), t>0, \\ k\left(\frac{\partial\left(c_{p} T\right)}{\partial x}-\frac{R T}{x}\right)=0, & x=0,1, t>0, \\ T(x, 0)=T_{0}(x), & x \in(0,1) .\end{cases}
$$

Problem (1.2) was raised in Stenchikov and Robock [13] (see also Gierasch and Goody [8] and Ramaswamy and Kielh [12]). We recall that $x$ represents the vertical coordinate in terms of the ratio $\frac{p}{p_{s}}$ between the air pressure to different heights and the surface air pressure (so $x=0$ corresponds to the top of the atmosphere and $x=1$ to the surface). In (1.2) $c_{p}(x) T(x, t)$ corresponds to the total specific energy in a dry convection situation: i.e. $u(x, t):=H(x, t)=c_{p}(x) T(x, t)$ with $c_{p}(x)>0$ the specific heat of air at constant pressure and $T(x, t)$ the temperature (we recall that the water vapor mixing ratio $q(x, t)$ is here assumed to be zero). The convective diffusion function $k(s)$ is assumed to be zero on "stable regions", i.e. when acting on nonpositive balances

$$
k\left(\frac{\partial u(x, t)}{\partial x}-\frac{R}{x c_{p}(x)} u(x, t)\right) \leq 0 .
$$

In fact, problem (1.2) corresponds to the general case $c_{p}(x)$ but the mathematical difficulties are the same than for $c_{p}=1$. 
One of the main difficulty, in the mathematical treatment of the problem (1.2) comes from the fact that the function $k$ is not "coercive", that is, it does not satisfy the condition

$$
\lim _{|s| \rightarrow \infty} k(s)=+\infty
$$

In the best of our knowledge, most of the results in the literature on the existence of solutions of quasilinear parabolic problems of the type (1.1) require the above coercivity assumption (see e.g. Ladyzhenskaya, Solonnikov and Uraltseva [9], Attouch and Damlamian [1], Benilan [3], etc.). A different problem with a non coercive formulation was considered in Díaz [6] Our treatment will make a fundamental use of the fact that the spatial variable is one-dimensional. This allows to show that the abstract theory of accretive operators can be applied in a suitable Banach space, which yields to the existence and uniqueness of a weak solution of (1.2).

\section{On the existence and uniqueness of the weak so- lution.}

In order to state the main result of this paper we start by introducing some functional spaces which are motivated by the presence at the equation of the singular term $\frac{R u}{x c_{p}(x)}$. Let $\Omega=(0,1)$, given $R>0$ we define the spaces

$$
L_{\rho}^{2}(\Omega)=\left\{f \in L_{l o c}^{1}(\Omega) ; \int_{\Omega} \rho(x) f^{2}(x) d x<\infty\right\}
$$

and

$$
V(\Omega)=\left\{f \in L_{\rho}^{2}(\Omega), \frac{\partial f}{\partial x} \in L_{\rho}^{2}(\Omega)\right\}
$$

where

$$
\rho(x)=e^{\int_{x}^{1} \frac{R}{\sigma c_{p}(\sigma)} d \sigma}
$$

Notice that $\rho(x)$ goes to infinity when $x$ goes to 0 . It is not difficult to show that those spaces become Hilbert spaces with the respective interior product

$$
<f, g>_{L_{\rho}^{2}(\Omega)}=\int_{\Omega} f(x) g(x) \rho(x) d x
$$

and

$$
<f, g>_{V(\Omega)}=<f, g>_{L_{\rho}^{2}(\Omega)}+<\frac{\partial f}{\partial x}, \frac{\partial g}{\partial x}>_{L_{\rho}^{2}(\Omega)}
$$

We have

Theorem 2.1 For any $u_{0} \in L_{\rho}^{2}(\Omega)$ there exists a unique mild solution $u \in C([0, T]$ : $\left.L_{\rho}^{2}(\Omega)\right)$ of problem (1.2). Moreover, if in addition we assume $u_{0} \in H_{\text {loc }}^{2}(\Omega)$ then the mild solution $u$ verifies that $u \in W^{1, \infty}\left(0, T: L_{\rho}^{2}(\Omega)\right)$ and $u$ is a strong solution of problem (1.2). 
We recall that given a Banach space $X$ and an abstract Cauchy problem

$$
\left\{\begin{array}{l}
\frac{d u(t)}{d t}+A u(t) \ni f(t), \quad t \in(0, T), \\
u(0)=u_{0},
\end{array}\right.
$$

where $A: D(A) \subset X \longrightarrow 2^{X}$ and $f \in L^{1}(0, T ; X)$, we say that $u:[0, T] \longrightarrow X$ is a mild solution of problem $(2.1)$ on $[0, T]$ if: $u \in C([0, T] ; X)$ and for each $\epsilon>0$ there exists at least one $\epsilon$-discretization $D_{A}\left(\epsilon, t_{0}, \ldots, t_{n} ; f_{1}, \ldots, f_{n}\right)$ of $(2.1)$ on $[0, T]$ (i.e. $0 \leq$ $t_{0} \leq t_{1} \leq t_{2}, \ldots, t_{n} \leq T ; \quad f_{1}, f_{2}, \ldots, f_{n} \in X$

$$
\left.t_{i}-t_{i-1} \leq \epsilon, \quad \forall i=1,2 \ldots n, \text { and } \sum_{i=1}^{n} \int_{t_{i-1}}^{t_{i}}\left\|f(s)-f_{i}\right\| d s \leq \epsilon\right),
$$

and a piecewise constant solution $v:\left[t_{0}, t_{n}\right] \longrightarrow X$ of the discretized implicit scheme

$$
\frac{v\left(t_{i}\right)-v\left(t_{i-1}\right)}{t_{i}-t_{i-1}}+A\left(v\left(t_{i}\right)\right) \ni f_{i},
$$

associated to $D_{A}\left(\epsilon, t_{0}, t_{1}, t_{2}, \ldots, t_{n} ; f_{1}, f_{2}, \ldots, f_{n}\right)$ on $[0, T]$ and such that

$$
\|u(t)-v(t)\| \leq \epsilon
$$

for each $t \in\left[t_{0}, t_{n}\right]$.

The function $u$ is a strong solution if $u(t) \in D(A)$ a.e. $t \in(0, T), u \in W_{l o c}^{1,1}(0, T$ : $X)$ and there exists $g \in L_{l o c}^{1}(0, T: X), g(t) \in A u(t)$ a.e. $t \in(0, T)$ such that

$$
\frac{d u}{d t}(t)+g(t)=f(t) \text { a.e. } t \in(0, T) .
$$

The proof of the Theorem 2.1 is based in the application of the abstract Crandall - Ligget Theorem for m-accretive operators, (see Crandall - Ligget [1971]).

We shall show that the results of Crandall -Ligget Theorem can be applied to the operator $A$ defined on the space $X=L_{\rho}^{2}(\Omega)$ by

$$
\begin{gathered}
A: D(A) \longrightarrow L_{\rho}^{2}(\Omega) \\
D(A)=\left\{v \in V(\Omega) ; \frac{\partial}{\partial x} k\left(\frac{\partial v}{\partial x}-\frac{R v}{x c_{p}(x)}\right) \in L_{\rho}^{2}(\Omega), k\left(\frac{\partial v}{\partial x}-\frac{R v}{x c_{p}(x)}\right)=0 \quad \text { on } \partial \Omega\right\}, \\
A(v)=-\frac{\partial}{\partial x} k\left(\frac{\partial v}{\partial x}-\frac{R v}{x c_{p}(x)}\right) \text { if } v \in D(A) .
\end{gathered}
$$

Lemma 2.1 $\overline{D(A)}=L_{\rho}^{2}(\Omega)$.

Proof. First, let us observe that $C_{c}^{\infty}(\Omega) \subset D(A)$. If $u \in C_{c}^{\infty}(\Omega)$ then $\rho(x) \frac{\partial u}{\partial x} \in$ $C_{c}^{\infty}(\Omega)$. Thus $\rho(x)^{-1} \frac{\partial}{\partial x}\left(\rho(x) \frac{\partial u}{\partial x}\right) \in C_{c}^{\infty}(\Omega) \subset L_{\rho}^{2}(\Omega)$. Now, let $u \in L_{\rho}^{2}(\Omega)$. Then $\rho(x)^{\frac{1}{2}} u \in L^{2}(\Omega)$, and since $\overline{C_{c}^{\infty}(\Omega)}=L^{2}(\Omega)$ there exists a sequence $u_{n} \in C_{c}^{\infty}(\Omega)$ such that $u_{n} \longrightarrow \rho(x)^{\frac{1}{2}} u$ in $L^{2}(\Omega)$. So we deduce that $\rho(x)^{-\frac{1}{2}} u_{n} \longrightarrow u$ in $L_{\rho}^{2}(\Omega)$ but $u_{n} \in C_{c}^{\infty}(\Omega)$ implies that $v_{n}=\rho(x)^{-\frac{1}{2}} u_{n} \in C_{c}^{\infty}(\Omega)$, and so $v_{n} \longrightarrow u$ in $L_{\rho}^{2}(\Omega)$. 
Lemma $2.2 A$ is accretive in $L_{\rho}^{2}(\Omega)$.

Proof. Let $u, v \in D(A)$ and $\lambda \geq 0$. Then

$$
\begin{gathered}
\|u-v+\lambda(A(u)-A(v))\|_{L_{\rho}^{2}(\Omega)}= \\
\left(\|u-v\|_{L_{\rho}^{2}(\Omega)}^{2}+\lambda^{2}\|(A(u)-A(v))\|_{L_{\rho}^{2}(\Omega)}^{2}+2 \lambda \int_{\Omega} \rho(x)(A(u)-A(v))(u-v) d x\right)^{\frac{1}{2}} \geq \\
\left(\|u-v\|_{L_{\rho}^{2}(\Omega)}^{2}-2 \lambda \int_{\Omega}\left(\frac{\partial}{\partial x}\left(k\left(\frac{\partial u}{\partial x}-\frac{R u}{x c_{p}(x)}\right)-k\left(\frac{\partial v}{\partial x}-\frac{R v}{x c_{p}(x)}\right)\right)(u-v) \rho(x) d x\right)^{\frac{1}{2}}=\right. \\
\left(\|u-v\|_{L_{\rho}^{2}(\Omega)}^{2}+2 \lambda \int_{\Omega}\left(k\left(\frac{\partial u}{\partial x}-\frac{R u}{x c_{p}(x)}\right)-k\left(\frac{\partial v}{\partial x}-\frac{R v}{x c_{p}(x)}\right)\right) \frac{\partial}{\partial x}(\rho(x)(u-v)) d x\right)^{\frac{1}{2}}= \\
\left(\|u-v\|_{L_{\rho}^{2}(\Omega)}^{2}+2 \lambda \int_{\Omega}\left(k\left(\frac{\partial u}{\partial x}-\frac{R u}{x c_{p}(x)}\right)-\right.\right. \\
\left.\left.k\left(\frac{\partial v}{\partial x}-\frac{R v}{x c_{p}(x)}\right)\right)\left(\left(\frac{\partial u}{\partial x}-\frac{R u}{x c_{p}(x)}\right)-\left(\frac{\partial v}{\partial x}-\frac{R v}{x c_{p}(x)}\right)\right) \rho(x) d x\right)^{\frac{1}{2}} .
\end{gathered}
$$

Since $k$ is a nonincreasing function and $k(0)=0$ we obtain that

$\left.\lambda \int_{\Omega}\left(k\left(\frac{\partial u}{\partial x}-\frac{R u}{x c_{p}(x)}\right)-k\left(\frac{\partial v}{\partial x}-\frac{R v}{x c_{p}(x)}\right)\right)\left(\left(\frac{\partial u}{\partial x}-\frac{R u}{x c_{p}(x)}\right)-\left(\frac{\partial v}{\partial x}-\frac{R v}{x c_{p}(x)}\right)\right) \rho(x) d x\right) \geq 0$

and then

$$
\|u-v+\lambda(A(u)-A(v))\|_{L_{\rho}^{2}(\Omega)} \geq\|u-v\|_{L_{\rho}^{2}(\Omega)}
$$

for each $\lambda \geq 0$.

The main difficulty of the proof of Theorem 1 arises in the proof of

Lemma $2.3 A$ is m-accretive in $L_{\rho}^{2}(\Omega)$

We introduce the subspace $V_{0}(\Omega) \subset V(\Omega)$ given by

$$
V_{0}(\Omega)={\overline{C_{0}}}^{V(\Omega)} \text {. }
$$

It is easy to see that $V_{0}(\Omega)$ coincides with the set $\{f \in V(\Omega), f=0$ on $\partial \Omega\}$ once that the trace of a function $f \in V(\Omega)$ is introduced in an usual manner (see e.g. Evans [7]). We also recall that given $f \in L_{\rho}^{2}(\Omega)$, a function $u \in D(A)$ verifies (in a weak sense) the equation $\lambda A u+u=f$ if, and only if,

$$
\begin{gathered}
\int_{\Omega} \rho(x) \psi u d x+\lambda \int_{\Omega} \rho(x) \psi\left(\frac{d}{d x}\left(k\left(\frac{d u}{d x}-\frac{R u}{x c_{p}(x)}\right)\right) d x-\int_{\Omega} \psi f \rho(x) d x=0\right. \\
\forall \psi \in L_{\rho}^{2}(\Omega) .
\end{gathered}
$$

Let $\beta: D(\beta) \longrightarrow \mathcal{P}(\mathbb{R})$ be the maximal monotone graph of $\mathbb{R}^{2}$ given as $\beta=k^{-1}$ i.e.

$$
\beta(s)= \begin{cases}s & \text { if } s>0 \\ (-\infty, 0) & \text { if } s=0 \\ \emptyset & \text { if } s<0\end{cases}
$$


Notice that $s \in \beta(k(s))$ and $r \in k(\beta(r))$, for any $s, r \in \mathbb{R}$. We denote by $j$ the primitive of $\beta$, such that $j(0)=0$, i.e.

$$
j(s)= \begin{cases}\frac{1}{2} s^{2} & \text { if } s \geq 0 \\ +\infty & \text { if } s<0 .\end{cases}
$$

Finally, we introduce the function $J: V(\Omega) \longrightarrow(-\infty,+\infty]$ given by

$$
J(v)= \begin{cases}\int_{\Omega} \rho(x) j(v) d x+\frac{1}{2} \int_{\Omega} \rho(x)\left(\frac{d v}{d x}\right)^{2} d x-\int_{\Omega} \rho(x) f \frac{d v}{d x} d x, & \text { if } j(v) \in L_{\rho}^{2}(\Omega), \\ +\infty, & \text { otherwise. }\end{cases}
$$

We have

Lemma $2.4 J$ is a proper, lower semicontinuous and coercive function on $V(\Omega)$.

Proof. $J$ is proper (i.e. $\exists u \in V(\Omega)$ such that $J(u)<+\infty$ ). Indeed, we can take $u=0$ and as $J(0)=0$ the conclusion is obvious.

$J$ is coercive in $V(\Omega)$ : (i.e. $J(u) \longrightarrow+\infty$ if $\left.\|u\|_{V(\Omega)} \longrightarrow+\infty\right)$. From the construction of $J$ it result that:

$$
J(u) \geq \frac{1}{2}\|u\|_{V}^{2}-\|u\|_{V}\|f\|_{L_{\rho}^{2}}
$$

and so we get the conclusion.

$J$ is lower semicontinuous in $V(\Omega)$ : Let $u_{n} \in V$ such that $u_{n} \rightarrow u$ strongly in $V(\Omega)$. If the lower limit of $J\left(u_{n}\right)$ is equal to infinity then, obviously

$$
\lim _{n \rightarrow \infty} J\left(u_{n}\right) \geq J(u)
$$

So, let us suppose that $I<\infty$, where $I=\lim \inf J\left(u_{n}\right)$. Then there exists a subsequence, that we denote again by $u_{n}$, such that $J\left(u_{n}\right) \rightarrow I$. Moreover $\rho(x)^{\frac{1}{2}} u_{n} \geq 0$ a.e. and $\left\|\rho(x)^{\frac{1}{2}} u_{n}\right\|_{L^{2}(\Omega)} \leq 2 I, \quad \forall n \geq n_{0}$

$$
\int_{\Omega} \rho(x) k\left(u_{n}\right)^{2} d x=\int_{\Omega} \rho(x) u_{n}^{2} d x .
$$

By the Lebesgue dominated convergence theorem we have that

$$
\int_{\Omega} u_{n}^{2} \rho(x) d x \geq \int_{\Omega}(u)^{2} \rho(x) d x=\int_{\Omega} k(u)^{2} \rho(x) d x,
$$

moreover, since $u_{n} \rightarrow u$ weakly in $V(\Omega)$ we get that

$$
\left\|u_{n}\right\|_{V(\Omega)} \geq\|u\|_{V(\Omega)}
$$

So, in conclusion

$$
I=\lim \inf J\left(u_{n}\right) \geq J(u)
$$

which proves the result.

$J$ is convex: It suffices to remark that $J$ is the addition of two convex functions $\int_{\Omega} \rho(x) j(v) d x$ and $\frac{1}{2} \int_{\Omega} \rho(x)\left(\frac{d v}{d x}\right)^{2} d x$ and a linear function $-\int_{\Omega} \rho(x) f \frac{d v}{d x} d x$. 
Proof of Lemma 3. Since $J$ is a coercive, 1.s.c. and convex function, there exists a minimum $w \in V(\Omega)$ of $J$. Moreover $0 \in \partial J(w)$. So we obtain that there exists $b \in L_{\rho}^{2}(\Omega)$ with $b(x) \in \beta(u(x))$ a.e. $x \in(0,1)$, such that

$$
\begin{gathered}
\int_{\Omega} \rho(x) b v d x+\lambda \int_{\Omega} \rho(x)\left(\frac{d w}{d x}\right)\left(\frac{d v}{d x}\right) d x+\int_{\Omega} \rho(x) f \frac{d v}{d x} d x=0, \\
\forall v \in V_{0}(\Omega) .
\end{gathered}
$$

Now, if we define

$$
u(x)=\rho(x)^{-1} \int_{0}^{x} \rho(\sigma) \beta(w(\sigma)) d \sigma+c \rho(x)^{-1},
$$

with $c=-\int_{\Omega}\left(f+\rho(x)^{-1} \int_{0}^{x} \rho(\sigma) b(\sigma) d \sigma-\lambda \frac{d w}{d x}\right) d x$, it results

$$
\int_{\Omega} \rho(x) b(x) v(x) d x=\int_{\Omega} v(x) \frac{d}{d x}\left(\int_{0}^{x} \rho(\sigma) b(\sigma) d \sigma\right) d x
$$

and integrating by parts we get:

$$
\begin{gathered}
\int_{\Omega} v(x) \frac{d}{d x}\left(\int_{0}^{x} \rho(\sigma) b(\sigma) d \sigma+c\right) d x= \\
-\int_{\Omega} \frac{d v}{d x}\left(\int_{0}^{x} \rho(\sigma) b(\sigma) d \sigma+c\right) d x+\left.v \int_{1}^{x} \rho(\sigma) b(\sigma) d \sigma\right|_{x=0} ^{x=1}=I .
\end{gathered}
$$

Since $v=0$ on $\partial \Omega$ we obtain that

$$
I=-\int_{\Omega} \rho(x) \frac{d v}{d x} \rho(x)^{-1}\left(\int_{0}^{x} \rho(\sigma) b(\sigma) d \sigma+c\right) d x=-\int_{\Omega} \rho(x) \frac{d v}{d x} u d x
$$

and

$$
\begin{gathered}
\int_{\Omega} \frac{d w}{d x} \frac{d v}{d x} \rho(x) d x=\int_{\Omega} \rho(x) \frac{d v}{d x} \frac{d}{d x}(k(b)) d x= \\
=\int_{\Omega} \rho(x) \frac{d v}{d x} \frac{d}{d x}\left(k\left(\rho(x)^{-1} \rho(x) b\right)\right) d x= \\
=\int_{\Omega} \rho(x) \frac{d v}{d x} \frac{d}{d x}\left(k\left(\rho(x)^{-1} \frac{d}{d x}\left(\int_{0}^{x} \rho(\sigma) b(\sigma) d \sigma\right)\right)\right) d x= \\
=\int_{\Omega} \rho(x) \frac{d v}{d x} \frac{d}{d x}\left(k\left(\rho(x)^{-1} \frac{d}{d x} \rho(x) \rho(x)^{-1}\left(\int_{0}^{x} \rho(\sigma) b(\sigma) d \sigma\right)\right) d x=\right. \\
\int_{\Omega} \rho(x) \frac{d v}{d x} \frac{d}{d x}\left(k\left(\rho(x)^{-1} \frac{d}{d x}(\rho(x) u)\right)\right) d x=\int_{\Omega} \rho(x) \frac{d v}{d x} \frac{d}{d x}\left(k\left(\frac{d u}{d x}-\frac{R u}{x c_{p}(x)}\right)\right) d x
\end{gathered}
$$

and so

$$
-\int_{\Omega} \rho(x) \frac{d v}{d x} u d x+\lambda \int_{\Omega} \rho(x)\left(\frac{d v}{d x}\right)\left(\frac{d}{d x}\left(k\left(\frac{d u}{d x}-\frac{R u}{x c_{p}(x)}\right)\right) d x-\int_{\Omega} \rho(x) \frac{d v}{d x} f d x=0,\right.
$$


for any $v \in V_{0}$. Is easy to prove that any $\psi \in L_{\rho}^{2}(\Omega)$ can be expressed as $\frac{d v}{d x}$ for some $v \in V(\Omega),\left(v=\int_{0}^{x} \psi d x\right)$, and $v \in V \cap C^{\infty}(\bar{\Omega})$ can be written as $v_{0}+m \int_{0}^{x} \rho(x)^{-1} d x$ where $v_{0} \in V_{0}(\Omega)$ and $m \in \mathbb{R}$. Then

$$
\begin{aligned}
& -\int_{\Omega} m u d x+\lambda \int_{\Omega} m\left(\frac{d}{d x}\left(k\left(\frac{d u}{d x}-\frac{R u}{x c_{p}(x)}\right)\right) d x-\int_{\Omega} m f d x=\right. \\
& m\left(-\int_{\Omega} u d x+\lambda \int_{\Omega}\left(\frac{d}{d x}\left(k\left(\frac{d u}{d x}-\frac{R u}{x c_{p}(x)}\right)\right) d x-\int_{\Omega} f d x\right)=0\right.
\end{aligned}
$$

as consequence of the choice of the constant $c$. Adding both expressions we obtain:

$$
\begin{gathered}
-\int_{\Omega} \rho(x) \frac{d\left(v_{0}+m \int_{0}^{x} \rho(\sigma)^{-1} d \sigma\right)}{d x} u d x+ \\
\lambda \int_{\Omega} \rho(x)\left(\frac{d\left(v_{0}+m \int_{0}^{x} \rho(\sigma)^{-1} d \sigma\right)}{d x}\right)\left(\frac{d}{d x}\left(k\left(\frac{d u}{d x}-\frac{R u}{x c_{p}(x)}\right)\right) d x-\right. \\
\int_{\Omega} \rho(x) \frac{d\left(v_{0}+m \int_{0}^{x} \rho(\sigma)^{-1} d \sigma\right)}{d x} f d x=0
\end{gathered}
$$

for any $v \in V_{0}$, and any $m \in \mathbb{R}$. Then

$$
-\int_{\Omega} \rho(x) \frac{d v}{d x} u d x+\lambda \int_{\Omega} \rho(x)\left(\frac{d v}{d x}\right)\left(\frac{d}{d x}\left(k\left(\frac{d u}{d x}-\frac{R u}{x c_{p}(x)}\right)\right) d x-\int_{\Omega} \rho(x) \frac{d v}{d x} f d x=0,\right.
$$

for any $v \in V \cap C^{\infty}$ and since $\overline{V \cap C^{\infty}}=V$, by density, we get that

$$
-\int_{\Omega} \rho(x) \frac{d v}{d x} u d x+\lambda \int_{\Omega} \rho(x)\left(\frac{d v}{d x}\right)\left(\frac{d}{d x}\left(k\left(\frac{d u}{d x}-\frac{R u}{x c_{p}(x)}\right)\right) d x-\int_{\Omega} \rho(x) \frac{d v}{d x} f d x=0,\right.
$$

for any $v \in V$. In conclusion:

$$
-\int_{\Omega} \rho(x) \psi u d x+\lambda \int_{\Omega} \rho(x) \psi\left(\frac{d}{d x}\left(k\left(\frac{d u}{d x}-\frac{R u}{x c_{p}(x)}\right)\right) d x-\int_{\Omega} \rho(x) \psi f d x=0\right.
$$

for any $\psi \in L_{p}^{2}(\Omega)$. Moreover, since $k\left(\frac{d u}{d x}-\frac{R u}{x c_{p}(x)}\right)=w \in V_{0}$ we get that $k\left(\frac{d u}{d x}-\frac{R u}{x c_{p}(x)}\right)=0$ on $\partial \Omega$ which ends the proof.

\section{On the non increasing number of stable and unsta- ble regions for regular solutions}

In this section we study some qualitative results on the solution of the problem. Our main goal is the study of the stable and unstable regions. We will work with the function

$$
w=\frac{d u}{d x}-\frac{R u}{x c_{p}(x)}
$$


Assumed $u$ is regular enough, then $w$ satisfies the problem

$$
\begin{cases}\frac{\partial w}{\partial t}=\rho^{-1} \frac{\partial}{\partial x}\left(\rho \frac{\partial}{\partial x} k(w)\right), & x \in(0,1), t>0, \\ k(w)=0, & x=0, \text { and } x=1, t>0, \\ w(x, 0)=w_{0}(x), & x \in(0,1),\end{cases}
$$

at least in the weak sense that

$$
\int_{\Omega} \rho \frac{\partial w}{\partial t} \eta d x+\int_{\Omega} \rho\left(\frac{\partial \eta}{\partial x}\right)\left(\frac{\partial}{\partial x}(k(w))\right) d x=0
$$

for any $\eta \in V_{0}$.

From the point of view of the physic of the model, the function $w$, represents the differential of the total static energy, (in the dry case), and gives us information about the stable and unstable regions. The region where $w \leq 0$, corresponds to a stable vertical distribution of static energy with absence of convection (remember that in this region $k \equiv 0$ ). The stable regions are formed by for the set of points of the vertical column of air $(x \in[0,1])$ such that $w \leq 0$, and the unstable regions, where $w>0$. Notice that in this last case convection occurs mixing the air.

The main goal of this section is to prove that the number of stable and unstable regions is not increasing in time for regular solutions. First, we shall prove that the boundary between stable and unstable regions is a semicontinuous graph. In this section we assume, for simplicity the extra regularity, $w \in C^{1}(\Omega \times(0, \infty))$.

Definition 3.1 Let $\phi_{i}(t)$ be defined by

$$
\begin{gathered}
\phi_{1}(t)=\inf \{x \in[0,1], w(x, t)>0,\}, \\
\phi_{2}(t)=\sup \left\{x>\phi_{1}(t), \text { such that } w(s, t)>0, \forall s \in\left(\phi_{1}(t), x\right)\right\}, \ldots, \\
\phi_{2 n}(t)=\sup \left\{x>\phi_{2 n-1}(t), \text { such that } w(s, t)>0, \forall s \in\left(\phi_{2 n-1}(t), x\right)\right\}, \\
\phi_{2 n+1}(t)=\sup \left\{x>\phi_{2 n}, \text { such that } w(s, t) \leq 0, \forall s \in\left(\phi_{2 n}(t), x\right)\right\} .
\end{gathered}
$$

As in Cui and Friedman [5], we prove, in the next proposition, the low semicontinuity of $\phi_{1}$. The case of $\phi_{2 i+1}$ and the upper semicontinuity of $\phi_{2 i}$, is proved in same way.

Proposition $3.1 \phi_{1}:[0, T] \longrightarrow(0,1)$, is lower semicontinuous.

We shall start by proving a technical lemma in order to prove the Proposition. By simplicity in the notation, we denote $\phi:=\phi_{1}$.

Lemma 3.1 There exists a constant $C$ which depends only of $\left\|w_{t}\right\|_{L^{\infty}}$, such that, if $t_{2}>t_{1}, t_{2}-t_{1} \leq 1$ we obtain:

$$
\phi(t) \geq \min \left\{\phi\left(t_{1}\right), \phi\left(t_{2}\right)\right\}-C\left(t_{2}-t_{1}\right)^{\frac{1}{2}}, \quad \forall t \in\left[t_{1}, t_{2}\right] .
$$


Proof. We consider

$$
b=\left(t_{2}-t_{1}\right)^{\frac{1}{2}} C, \quad a=\min \left\{\phi\left(t_{1}\right), \phi\left(t_{2}\right)\right\}-b .
$$

If $a \leq 0$ the conclusion holds trivially. Assume $a>0$. We define the function

$$
\bar{w}(x)= \begin{cases}c \int_{a}^{a+x}(\sigma-a)^{-\frac{1}{3}} \rho^{-1}(\sigma) d \sigma, & \text { if } a \leq x \leq a+b \\ 0, & \text { if } x \leq a\end{cases}
$$

Function $\bar{w}$ is well defined (because $a>0$ and $\rho^{-1} \geq \alpha>0$, if $x>a$ ) and $\bar{w} \in C^{1}$. It results

$$
\begin{gathered}
\bar{w}(0, t)=0=k(w(0, t)) \geq w(0, t), \\
\bar{w}(a+b, t)=c \int_{a}^{a+b}(\sigma-a)^{-\frac{1}{3}} \rho^{-1}(\sigma) d \sigma \leq 3 c \rho^{-1}(a) b^{\frac{1}{3}},
\end{gathered}
$$

and, on the other hand,

$$
w(a+b, t)=w\left(a+b, t_{1}\right)+w_{t}(a+b, \tau)\left(t-t_{1}\right),
$$

for some $\tau \in\left(t_{1}, t\right)$. Since $\left\|w_{t}\right\|_{L^{\infty}} \leq C$ and $w\left(a+b, t_{1}\right)=0$, it results

$$
w(a+b, t) \leq\left\|w_{t}\right\|_{L^{\infty}}\left(t_{2}-t_{1}\right) \leq C\left(t_{2}-t_{1}\right)^{\frac{1}{3}}=b,
$$

consequently, we deduce that

$$
\bar{w}(a+b, t) \geq w(a+b, t) .
$$

By construction we have that,

$$
\frac{\partial \bar{w}}{\partial t}-\rho^{-1} \frac{\partial}{\partial x}\left(\rho \frac{\partial}{\partial x} k(\bar{w})\right)=\rho^{-1} \frac{k}{2}(x-a)^{-\frac{4}{3}} \geq 0 .
$$

by (3.3), (3.5) and (3.6). Since $\bar{w} \in C^{0}$ we obtain $\bar{w}$ is a supersolution of the problem (3.1). By the comparison principle, (or T-accretiveness in $L^{1}$ of the operator), it results

$$
\bar{w} \geq w, \quad \text { in }\left(t_{1}, t_{2}\right) \times(a, a+b) .
$$

Consequently $k(w)=0$ in $x \leq a, t_{1}<t<t_{2}$, which proves that

$$
\phi(t) \geq a=\min \left\{\phi\left(t_{1}\right), \phi\left(t_{2}\right)\right\}-C\left(t_{1}-t_{2}\right)^{\frac{1}{2}} .
$$

Proof of the Proposition 3.1. Let

$$
a_{1}=\liminf _{t \rightarrow t_{0}^{-}} \phi(t), \quad a_{2}=\limsup _{t \rightarrow t_{0}^{-}} \phi(t) .
$$

In order to show the lower semicontinuity of $\phi$ we shall assume that

$$
a_{1}<a_{2}
$$


and proceed to derive a contradiction. There exists an increasing subsequence $t_{n}$ such that

$$
\phi\left(t_{2 k}\right) \rightarrow a_{1} \text {, and } \phi\left(t_{2 k+1}\right) \rightarrow a_{2} .
$$

By Lemma 3.1 we know that

$$
\phi\left(t_{2 i}\right) \geq \min \left\{\phi\left(t_{2 i-1}, t_{2 i+1}\right\}-C\left(t_{2 i+2}-t_{2 i}\right)^{\frac{1}{2}} .\right.
$$

Taking limits when $i \longrightarrow \infty$ we obtain

$$
a_{1} \geq a_{2},
$$

which is a contradiction with (3.7). Then $a_{1}=a_{2}$.

In the same way, by taking a decreasing sequence $t_{k}$, we get that the existence of

$$
\lim _{t \rightarrow t_{0}^{+}} \phi(t) .
$$

We shall show now that

$$
\lim _{t \rightarrow t_{0}^{+}} \phi(t) \leq \lim _{t \rightarrow t_{0}^{-}} \phi(t) .
$$

We proceed assuming $\lim _{t \rightarrow t_{0}^{+}} \phi(t) \geq \lim _{t \rightarrow t_{0}^{-}} \phi(t)$ and we shall derive a contradiction. Let

$$
\lim _{t \rightarrow t_{0}^{+}} \phi(t)=a_{3} \text { and } \lim _{t \rightarrow t_{0}^{-}} \phi(t)=a_{1},
$$

where $a_{1}<a_{3}, w>0$ in the region

$$
Q_{1}=\left\{(x, t), a_{1}<x_{0}-\delta \leq x \leq x_{0}+\delta<a_{3}, t_{0}-\delta \leq t \leq t_{0}\right\},
$$

and $w\left(x, t_{0}\right)=0$ on $\left(x_{0}-\delta, x_{0}+\delta\right)$, for some positive $\delta$. Let $\Sigma$ be the parabolic boundary of $Q$. By the choice of $Q, w$ satisfies

$$
\left\{\begin{array}{lc}
\frac{\partial w}{\partial t}-\rho^{-1} \frac{\partial}{\partial x}\left(\rho \frac{\partial}{\partial x} w\right)=0, & \text { in } Q, \\
w(x, t)=\left.w(x, t)\right|_{\chi_{\Sigma}} \geq 0, & \text { on }(x, t) \in \Sigma,
\end{array}\right.
$$

and $w\left(x, t_{0}-\delta\right)>0$. We consider the problem

$$
\begin{cases}\frac{\partial \underline{w}}{\partial t}-\rho^{-1} \frac{\partial}{\partial x}\left(\rho \frac{\partial}{\partial x} \underline{w}\right)=0, & \text { in } Q, \\ \underline{w}(x, t)=0, & (x, t) \in \Sigma .\end{cases}
$$

Since $L_{\rho}^{2}(\Omega)$ is a Hilbert space, there exists a orthonormal basis of eigenfunctions $u_{n}$, such that

$$
-\rho^{-1} \frac{\partial}{\partial x}\left(\rho \frac{\partial}{\partial x} u_{n}\right)+\lambda_{n} u_{n}=0
$$

and the solution of the problem (3.9) is

$$
\underline{w}=\sum_{i=1, \infty} a_{n} e^{-\lambda_{n} t} u_{n}
$$


where

$$
a_{n}=\int_{x_{0}-\delta}^{x_{0}+\delta} \rho(x) u_{n} \underline{w}\left(x, t_{0}\right) d x .
$$

Since $w\left(x, t_{0}\right) \neq 0$ in $\left(x_{0}-\delta, x_{0}+\delta\right)$, there exists $n_{0}$ such that $a_{n_{0}} \neq 0$. Taking $u_{n_{0}}$ as test function in (3.9) it results

$$
\int_{x_{0}-\delta}^{x_{0}+\delta} \rho \frac{\partial \underline{w}}{\partial t} u_{n_{0}} d x+\lambda_{n_{0}} a_{n_{0}} \int_{x_{0}-\delta}^{x_{0}+\delta} \rho u_{n_{0}}^{2} d x=0,
$$

but, since $u_{n}$ is orthonormal, we get that

$$
\left[\lambda_{n_{0}} e^{\lambda_{n_{0}} t}+\lambda_{n_{0}}\right] a_{n_{0}}=0
$$

which is a contradiction with the choice of $n_{0}$. Then $a_{3}=a_{1}$, and furthermore the low semicontinuity of $\phi$ is proved. In the same way, we prove the upper semicontinuity of $\phi_{2 i}$.

Now we prove the main result of this section.

Theorem 3.1 The solution $u$ of the problem (1.1) does not increase the number of stable (respectively unstable) regions.

The first part of the proof is related to stable regions. Let $\Omega_{0}$ be a connected stable region of the interval $[0,1]$. This region changes with the time. Let $\Omega_{0}(t)$ be this region at time $t$, let $a(t)$ and $b(t)$ be the evolution of his boundary, (they can be 0 , or 1 ). By the regularity of the solution they are continuous functions. Then $\Omega_{0}(t)$ is connected for all $t>0$. We take $k(w)$ as test function. Integrating by parts in $\Omega_{0}(t)$ we obtain:

$$
\frac{1}{2 k_{0}} \int_{\Omega_{0}(t)} \rho(x) \frac{\partial k^{2}(w)}{\partial t} d x+\int_{\Omega_{0}(t)} \rho(x)\left(\frac{\partial}{\partial x}(k(w))\right)^{2} d x=0
$$

since $w(a(t))=w(b(t))=0$, and so

$$
\frac{1}{2 k_{0}} \int_{0}^{t^{*}} \int_{b(t)}^{a(t)} \rho(x) \frac{\partial\left(k^{2}(w)\right)}{\partial t} d x d t+\int_{0}^{t^{*}} \int_{b(t)}^{a(t)} \rho(x)\left(\frac{\partial}{\partial x}(k(w))\right)^{2} d x d t=0 .
$$

Applying Leibnitz Theorem,

$$
\frac{1}{2 k_{0}} \int_{0}^{t^{*}} \frac{\partial}{\partial t}\left(\int_{b(t)}^{a(t)} \rho(x) k^{2}(w) d x\right) d t+\int_{0}^{t^{*}} \int_{b(t)}^{a(t)} \rho(x)\left(\frac{\partial}{\partial x}(k(w))\right)^{2} d x d t=0
$$

Since $w(a(t))=w(b(t))=0$ and integrating by parts in $t$, it result that

$$
\frac{1}{2 k_{0}} \int_{\Omega_{0}\left(t^{+}\right)} \rho(x) k^{2}(w) d x-\frac{1}{2 k_{0}} \int_{\Omega_{0}} \rho(x) k^{2}(w) d x+\int_{0}^{t^{*}} \int_{b(t)}^{a(t)} \rho(x)\left(\frac{\partial}{\partial x}(k(w))\right)^{2} d x d t=0 .
$$

Since $\Omega_{0}$ is a stable region $\int_{\Omega_{0}\left(t^{*}\right)} \rho(x) k^{2}(w) d x=0$ for any $t^{*}>0$. Then does not appear new unstable regions in his interior and $\Omega_{0}\left(t^{*}\right)$ is connected.. In the second part of the proof, let $\Omega_{1}$ a connected unstable region, caused by the boundary conditions, $\Omega_{1}$ is in the inner of the interval, like in the stable regions. We denote by $\Omega_{1}(t)$ the evolution 
of this region, we proceed by reduction to the absurd. Let us suppose there exists a $t^{*}>0$ such that $\Omega_{1}\left(t^{*}\right)$ is not connected. We take an interval $(c, d) \subset(\min \{x \in$ $\left.\left.\Omega_{1}\left(t^{*}\right)\right\}, \max \left\{x \in \Omega_{1}\left(t^{*}\right)\right\}\right)$, such that there is a connected stable region enclosed in this. Let $t_{1}<t^{*}$ be such that $(c, d) \subset \Omega_{1}\left(t_{1}\right)$ and $c \in \Omega_{1}(t), d \in \Omega_{1}(t)$ for all $t \in\left[t_{1}, t^{*}\right]$ let $k_{1}=\min \left\{w\left(x, t_{1}\right), w(c, t), w(d, t)\right.$, with $x \in(c, d)$ and $\left.t \in\left[t_{1}, t^{*}\right]\right\}$. It is clear that $k_{1}$ is a positive constant. Let $s: \mathbb{R} \rightarrow \mathbb{R}$ be the positive part function. Let $s_{\varepsilon}$ a convex regularization of $s$ such that $s(\cdot)>s_{\varepsilon}(\cdot)$. Let $g_{\varepsilon}(t)=\int_{c}^{d} \rho(x) s_{\varepsilon}\left(k_{1}-w(x, t)\right) d x$. By the regularity of $w$ and $s_{\varepsilon}$, it results that $g_{\varepsilon}$ is a $C^{1}$ function. Differentiating the function we get that

$$
\frac{d g_{\varepsilon}(t)}{d t}=-\int_{c}^{d} \rho(x) \frac{\partial w}{\partial t} s_{\varepsilon}^{\prime}\left(k_{1}-w(x, t)\right) d x=-\int_{c}^{d} \rho(x) s_{\varepsilon}^{\prime \prime}\left(k_{1}-w(x, t)\right)\left(\frac{\partial}{\partial x}(k(w))^{2} d x .\right.
$$

From the convexity of the function it results that $\frac{d g_{\varepsilon}(t)}{d t} \leq 0$, since $g_{\varepsilon}(0)=0$, and $g_{\varepsilon}$ is a positive function we get $g_{\varepsilon}(t)=0$, that implies $w(x, t) \geq k_{1}$ for all $(x, t) \in(c, d) \times\left(t_{1}, t^{*}\right)$. But then there would not exists a stable region in the interior of $(c, d)$, which is a contradiction with the assumption of the existence. In conclusion a stable (unstable) region can disappear for a certain $t>0$, but it never may appear any stable (unstable) region.

\section{Acknowledgments}

The authors thank Professors L.G.Stenchikov and G. Hetzer for various discussions concerning this work which was partially supported by the DGES (Spain), project REN2000/0766.

\section{References}

[1] H.Attouch, A.Damlamian, Applications des methodes de convexité et monotonie à l'étude de certaines équations quasi lineáires, Proc. Roy. Soc. Edinburgh 79 (A) (1977) 107-129.

[2] V.Barbu, Nonlinear semigroups and differential equations in Banach spaces, Noordhoff International Publishing Romania, Romania 1976.

[3] Ph. Benilan, Equations d'évolution dans un espace de Banach quelconque et applications. Thèse, Orsay, 1972.

[4] M.G.Crandall, T.M.Ligget, Generation of semi-groups of nonlinear transformation on general Banach spaces, Amer. J. Math. 93 (1971) 265-298.

[5] S.Cui and A.Friedman, Manuscript, (personal communication 1999).

[6] J.I. Díaz, On a fully nonlinear parabolic equation and the asymptotic behaviour of its solutions, Journal of Math. Analysis and Application 95 (1) (1983) 144-168. 
[7] L.C.Evans, Application of nonlinear semigroup theory to certain partial differential equations. In M.G.Crandall (Ed), Proceedings on "Nonlinear evolution equations" Academic Press, N.Y. 1978, pp. 163-188.

[8] P.J. Gierasch, R. Goody, A study of the thermal and dynamical structure of the martian lower atmosphere. Planet Space Sci. 16 (1968) 615-646.

[9] O.H.Ladyzenkaja, V.A.Solonnikov, N.N.Uralceva, Linear and quasi-linear equations of parabolic type, Translations of Mathematical monographs 23, Amer. Math. Soc. Providence 1968.

[10] S.Manabe, R.F. Strickler, Thermal equilibrium of the atmosphere with a convective adjustment, J. Atmos Sci. 21 (1964) 361-365.

[11] H.Matano, Nonincrease of the lap-number of a solution for a one-dimensional semilinear parabolic equation, Journal of the Faculty of Science of the University of Tokio Sec IA 29 (1982) 401-441.

[12] V.Ramaswamy and J.T. Kielh, Sensitives of the radiative forcing due to large losdings of smoke and dust aerosols, J. Geophys. Res. 90 (1985) 5597-5613.

[13] L.G. Stenchikov, A. Robock, Diurnal asymmetry of climatic response to increased $\mathrm{CO}_{2}$ and aerosols: Forcing and feedbacks, Journal of Geophisical Res. 100 (D12) (1995) 26211-26227.

[14] L.G.Stenchikov, A.Robock, Climatic effects due to water vapor amount increase in the stratosphere after the Pinatubo eruption, EOS Trans. AGU, (Fall Meet. Suppl.) 74 (43) (1993) 114.

[15] J.I. Tello, Tratamiento Matemático de Procesos Físicos con Difusión y Convección. Ph.D. Thesis, Universidad Complutense de Madrid, Madrid 2001. 